\title{
1.2-km Timing-Stabilized, Polarization-Maintaining Fiber Link with Sub-Femtosecond Residual Timing Jitter
}

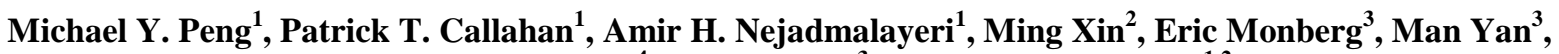 \\ Lars Grüner-Nielsen ${ }^{4}$, John M. Fini ${ }^{3}$ and Franz X. Kärtner ${ }^{1,2}$ \\ ${ }^{1}$ Department of Electrical Engineering and Computer Science and Research Laboratory of Electronics, \\ Massachusetts Institute of Technology, 77 Massachusetts Ave., Cambridge, Massachusetts 02139, USA \\ ${ }^{2}$ Center for Free-Electron Laser Science, DESY and Department of Physics, University of Hamburg, Notkestraße 85, Hamburg 22607, Germany \\ ${ }^{3}$ OFS Laboratories, 25 Schoolhouse Road, Somerset, New Jersey 08873, USA \\ ${ }^{4}$ OFS, Priorparken 680, DK-2605 Brondby, Denmark \\ Author e-mail address: mypeng@mit.edu
}

\begin{abstract}
A 1.2-km timing-stabilized, polarization-maintaining fiber link based on balanced optical cross-correlation was demonstrated with $\sim 0.9$ fs RMS timing jitter over 16 days and $\sim 0.2$ fs RMS timing jitter over 3 days.

OCIS codes: (060.2360) Fiber optics links and subsystems; (120.4825) Optical time domain reflectometry; (320.7160)

Ultrafast technology
\end{abstract}

\section{Introduction}

Femtosecond performance for long-distance and long-term timing distribution is critical for realizing the full potential of next-generation X-ray free-electron lasers (X-FEL). Today, these short-pulse X-ray sources require tight synchronization between lasers and RF sources across $300 \mathrm{~m}$ to $3 \mathrm{~km}$ distances with less than $10 \mathrm{fs}$ precision. Due to the rapid progress in X-FEL technology, sub-fs-level timing distribution will be demanded in the years to come.

To address these demands, we have developed an ultrafast, fiber-optic, timing distribution system based on balanced optical cross-correlation [1]. The timing signal, which is an ultralow-noise optical pulse train generated from a mode-locked laser [2], is distributed via a fiber-optic link. When the link is not stabilized, slow fiber fluctuations due to thermal and acoustical disturbances, for example, will introduce timing errors in the output pulse arrival times. By using a partial reflector at the link output, the timing error can be measured directly at the link input using a balanced optical cross-correlator (BOC). The BOC is based on balanced detection of a double-pass, type-II phase-matched second harmonic generation (SHG) between a reflected output pulse and a new pulse from the laser source. The error voltage signal, which is directly proportional to the timing error, is fed back to a free-space delay to correct for the fiber fluctuations. Our previous work on a 300-m SMF link was limited to about $10 \mathrm{fs}$ of stability due to polarization-mode dispersion, which is known to induce up to $100 \mathrm{fs}$ of long-term drift if left unmanaged [3-4]. Improving upon these results, we demonstrate here a 1.2-km polarization-maintaining, dispersion-compensated fiber link with sub-fs residual timing jitter for over 16 days of uninterrupted operation.

\section{Experimental Set-up}

The experimental set-up of the timing-stabilized PM link is shown in Figure 1a. The 200-MHz repetition rate laser source is a free-running Er-doped fiber laser (Menlo Systems) that outputs 160-fs pulses centered at $1560 \mathrm{~nm}$ with $+20 \mathrm{dBm}$ average power. The pulse width and repetition rate are selected to keep higher-order dispersion and fiber nonlinearity low. The set-up is divided into two sections: the in-loop, which is responsible for link stabilization, and the out-of-loop, which is used to measure the in-loop performance.

In the in-loop section, the pulses are first divided into a reference and signal path. The reference path serves as a timing reference for the in-loop BOC. The signal path consists of a voltage-controlled free-space delay, polarization optics, 1.2-km PM link, and 50/50 output coupler. Since the forward and reflected pulses must propagate on the same polarization axis in the PM link, the $45^{\circ}$ Faraday rotator is necessary to induce a $90^{\circ}$ round-trip polarization rotation so that the reflected pulses will reach the in-loop BOC. The half-wave plate aligns the free-space polarization with the slow axis of the PM fiber. The 1.2-km PM link, which was designed and manufactured in collaboration with OFS, contains $1082 \mathrm{~m}$ of standard PM fiber and $190 \mathrm{~m}$ of dispersion-compensated PM fiber (PM-DCF). The PM-DCF has dispersion and dispersion slope of $-104.1 \mathrm{ps} /(\mathrm{nm} \cdot \mathrm{km})$ and $-0.34 \mathrm{ps} /\left(\mathrm{nm}^{2} \cdot \mathrm{km}\right)$, respectively, at $1550 \mathrm{~nm}$. Residual link dispersion of $-0.06 \mathrm{ps} / \mathrm{nm}$ at $1560 \mathrm{~nm}$ was compensated for by adding $2.4 \mathrm{~m}$ of standard PM fiber. The coupled input power to the PM link was $+11 \mathrm{dBm}$, which is near the onset of fiber nonlinearities. With a 50/50 output coupling ratio, the reflected power was sufficient for in-loop stabilization without the use of an EDFA. The timing error is detected with the in-loop BOC, which consists of a single 4-mm PPKTP crystal operated in a double-pass configuration with appropriate dichroic elements [3]. The timing sensitivity was measured to be $\sim 21 \mathrm{mV} / \mathrm{fs}$. The stabilization feedback loop consists of a PI controller (Menlo, PIC210 Lockbox), high voltage amplifier (Menlo, HVA150) and a stacked combination of a 40- $\mu$ m piezoelectric actuator (Thorlabs, PAS009) and a 25-mm motor translation stage (PI, M-112.12S) for short- and long-term stabilization, respectively. The feedback bandwidth was about $20 \mathrm{~Hz}$. 
The out-of-loop BOC, which is used to evaluate the in-loop stabilization, monitors the timing error between the link output pulses and new pulses from the laser. The timing sensitivity of the out-of-loop BOC was measured to be $\sim 2.3 \mathrm{mV} / \mathrm{fs}$. For long-term drift measurements, the out-of-loop voltage is logged on a CPU with an A/D converter (National Instrument, cDAQ-9172) at a $1 \mathrm{~Hz}$ sampling rate. A low-pass RC filter with a $0.5-\mathrm{Hz}$ bandwidth was used for anti-aliasing. The position of the motor translation stage was also recorded simultaneously.

Temperature stability is critical for sub-femtosecond-level performance. Temperature-stabilized enclosures were built separately for the free-space optics and the PM fiber link. The enclosures consisted of a 2" insulation foam and an internal Aluminum enclosure, which was actively temperature controlled with a resistive heater and PID temperature controller. Both the free-space optics and link enclosures had a constant setpoint of $27^{\circ} \mathrm{C}$ and were configured to be as resistant as possible to ambient temperature fluctuations. In addition, the link temperature was modulated with a period of $20 \mathrm{~min}$ and peak deviation of $\sim 0.05^{\circ} \mathrm{C}$ to aid in decoupling temperature-dependent effects between the two enclosures.
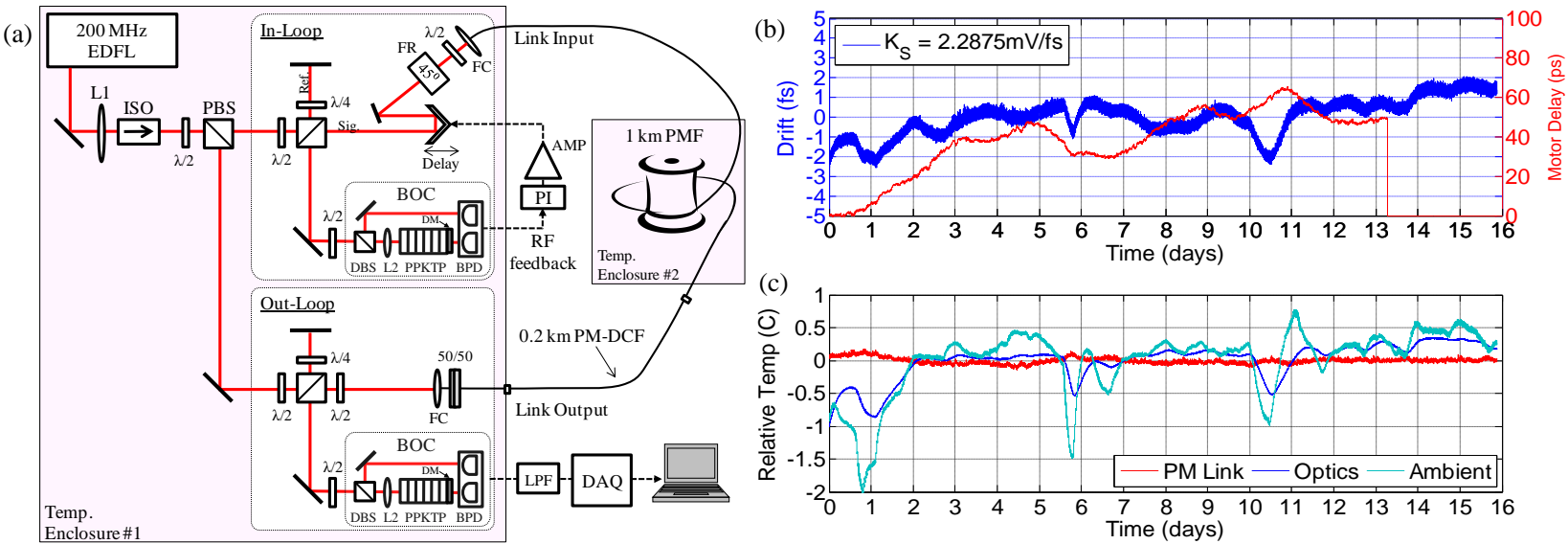

Figure 1a) Schematic of the timing-stabilized PM fiber link. (EDFL, Erbium-doped fiber laser; L1, collimator; ISO, isolator; $\lambda / 2$, half-wave plate; $\lambda / 4$, quarter-wave plate; PBS, polarizing beam-splitter; FR, $45^{\circ}$ Faraday rotator; FC, fiber collimator; PMF, standard PM fiber; PM-DCF, dispersion-compensated PM fiber; 50/50, output coupler; BOC, balanced optical cross-correlator; DBS, dichroic beam-splitter; L2, focusing lens; PPKTP, periodically-poled $\mathrm{KTiOPO}_{4}$; DM, dichroic mirror; $\mathrm{BPD}$, balanced photodetector; PI, proportional-integral controller; AMP, high voltage amplifier; LPF, low-pass filter; DAQ, A/D converter. 1b) Long-term timing drift measurements over 16 days of uninterrupted operation; includes out-of-loop drift (as measured by the BOC), and motor translation stage position. 1c) Corresponding long-term temperature drift measurements; includes internal temperatures of the PM link and free-space optics enclosures and the external ambient temperature.

\section{Results and Conclusion}

Link stabilization with uninterrupted operation for over 16 days yielded $\sim 0.9$ fs of RMS timing jitter. The out-of-loop drift, motor delay, and temperature measurements are shown in Figure $1 \mathrm{~b}$ and $\mathrm{c}$. The data acquisition for the motor stage accidentally faulted in day 13, but the link stabilization remained uninterrupted. With over $65 \mathrm{ps}$ $(19.5 \mathrm{~mm})$ peak-to-peak fiber length fluctuations, our link stabilization scheme successfully suppressed timing error by over four orders of magnitude to $3.5 \mathrm{fs}(1.0 \mu \mathrm{m})$ peak-to-peak. Under closer inspection, the major fluctuations in link drift is highly correlated with the ambient temperature; this is prominent on weekends when the $\mathrm{A} / \mathrm{C}$ settings in the lab were relaxed. Ambient temperature fluctuations only affect the free-space optics enclosure because of its large volume and non-uniform heating compared to the PM link enclosure. For days 11-14 when the ambient temperature fluctuations are fortuitously minimal, the 3-day operation yielded $\sim 0.2$ fs of RMS timing jitter.

Since the measured drift is believed to originate from temperature-dependent length fluctuations in the free-space optics, we have not yet observed the full potential of this PM link stabilization scheme. Ongoing work is focused on improving temperature stability of the BOCs and switching to an all-fiber implementation. This effort requires the development of an all-integrated, fiber-coupled PPKTP BOC [5].

\section{References}

[1] J. Kim, J. A. Cox, J. Chen, and F. X. Kärtner, "Drift-free femtosecond timing synchronization of remote optical and microwave sources," Nat Photon, vol. 2, no. 12, pp. 733-736, Dec. 2008.

[2] H. A. Haus and A. Mecozzi, "Noise of mode-locked lasers," Quantum Elec., IEEE Journal of, vol. 29, no. 3, pp. 983-996, Mar. 1993.

[3] J. Kim, J. Chen, Z. Zhang, F. N. C. Wong, F. X. Kärtner, F. Loehl, and H. Schlarb, "Long-term femtosecond timing link stabilization using a single-crystal balanced cross correlator," Opt. Lett., vol. 32, no. 9, pp. 1044-1046, May 2007.

[4] J. A. Cox, J. Kim, J. Chen, and F. X. Kärtner, "Long-term stable timing distribution of an ultrafast optical pulse train over multiple fiber links with polarization maintaining output," in Proc. of CLEO/QELS 2009, Baltimore, MD.

[5] A. H. Nejadmalayeri, F. N. C. Wong, T. D. Roberts, P. Battle, and F. X. Kärtner, "Guided wave optics in periodically poled KTP: quadratic nonlinearity and prospects for attosecond jitter characterization,” Opt. Lett., vol. 34, no. 16, pp. 2522-2524, 2009. 